\title{
High directivity modes in the Koch island fractal patch antenna
}

\author{
Jordi Romeu, Carmen Borja, Sebastian Blanch \\ Department of Signal Theory and Communications, Universitat Politecnica de \\ Catalunya \\ Jordi Girona 1-3, 08034 Barcelona,SPAIN romeu@tsc.upc.es
}

1 Introduction The use of fractal geometries in the design of antennas has been a subject of much attention. The self-similarity properties of certain fractals result in a multiband behavior of the antennas built after these fractals [1]. On the other hand, the high convoluted shape of these fractals makes possible the reduction in size of certain antennas [2]. Another interesting property of fractals are found in resonators with a fractal boundary. Some theoretical and experimental work on what is known as the "fractal drum" show that certain high order vibrational modes of a membrane with a fractal boundary exhibit a localization property [3]. These vibrational modes, known as fractinos, have the interesting property of localizing the vibration in small areas of the membrane. As a first approximation there are similarities in the behavior of a vibrating membrane and a microstrip patch antenna. When the cavity model for the microstrip patch antenna is considered in both cases the solution is derived from the eigenfunctions of the Helmholtz equation. Different boundary conditions are considered in each case. The Dirichlet boundary condition for the vibrating membrane and the Neumann boundary condition for the microstrip fractal antenna. The solution of the Helmholz equation with the Neumann boundary condition exhibit fractinos that are localized near the boundary [4]. These Neumann fractinos in a microstrip patch antenna would result in high currents located at the edges of the antenna. As long as these currents are in phase a broadside radiation pattern for the antenna is expected. These current distribution must result in a directive pattern, since these modes occur at a resonant frequency above the fundamental. Therefore, the size of the patch is larger than half a wavelength. As an example of this phenomenon experimental data on the Koch island patch antenna is presented.

\section{The Koch island patch antenna}

The Koch island patch antenna geometry is shown in Fig. 1. A three iteration version of the island has been built. The island has been etched on a $0.8 \mathrm{~mm}$ substrate with $\epsilon_{r}=3.38$. An additional air gap of $2.7 \mathrm{~mm}$ between the substrate and the ground plane is considered. The patch is fed through a coaxial probe.

3 Results The measured input impedance at two resonant frequencies is shown in Fig. 2. The fundamental frequency corresponds to $1.26 \mathrm{GHz}$. At the fundamental frequency the input impedance is well matched to $50 \Omega$ at the chosen feeding point. At the frequency of $4.04 \mathrm{GHz}$ an upper mode is excited. The impedance shows an 
inductive behavior, probably due to the inductive effect of the feeding probe. At this higher frequency this effect is more evident than at $1.26 \mathrm{G} \mathrm{Hz}$. On the other hand, the feeding point for this upper mode is not optimized and the input resistance is lower than $50 \Omega$. In any case, there are different methods to match the antenna.

The measured radiation patterns at these two frequencies are shown in Fig. 3. At the fundamental frequency, a broad broadside pattern with a directivity of $9 d B$ is obtained. At the fundamental frequency the size of the patch is $\lambda / 2$, therefore a broad pattern is obtained. At the frequency of $4.04 \mathrm{GHz}$ a narrower broadside pattern is obtained. The directivity in this case is $13 d B$, that is $4 d B$ higher than at the fundamental frequency. At this frequency the size of the patch is $1.5 \lambda$. This also explains the observation of nulls in the pattern.

4 Conclusion It has been shown that a patch antenna with fractal boundary exhibits, at a frequencies above the fundamental mode, localized modes. These modes can have broadside directive patterns. Fractal boundary microstrip patch antennas are patent pending.

5 Acknowledgments This research has been financed by the European Commission and CICYT through grant FEDER 2FD97-0135.

\section{References}

[1] C. Puente, J. Romeu, R. Pous, A. Cardama. On the Behavior of the Sierpinski Multiband Antenna IEEE Transactions on Antennas and Propagation, vol. 46, no.4, pp.517-524, April 1998.

[2] Puente, C., Romeu, J., Pous, R., Ramis, J., Hijazo, A. Small but long Koch fractal monopole, IEE Electronics Letters, vol. 34, no. 1, pp.9-10, January 1998.

[3] Sapoval, B., Gobron, Th. Vibrations of strongly irregular or fractal resonators Physical Review E, vol. 47, no.5, pp.3013-3024, May 1993.

[4] Russ, S., Sapoval, B., Haeberle, O. Irregular and fractal resonators with Neumann boundary conditions: Density of states and localization Physical Review $E$, vol. 55, no. 2, pp. 1413-1421, February 1997. 


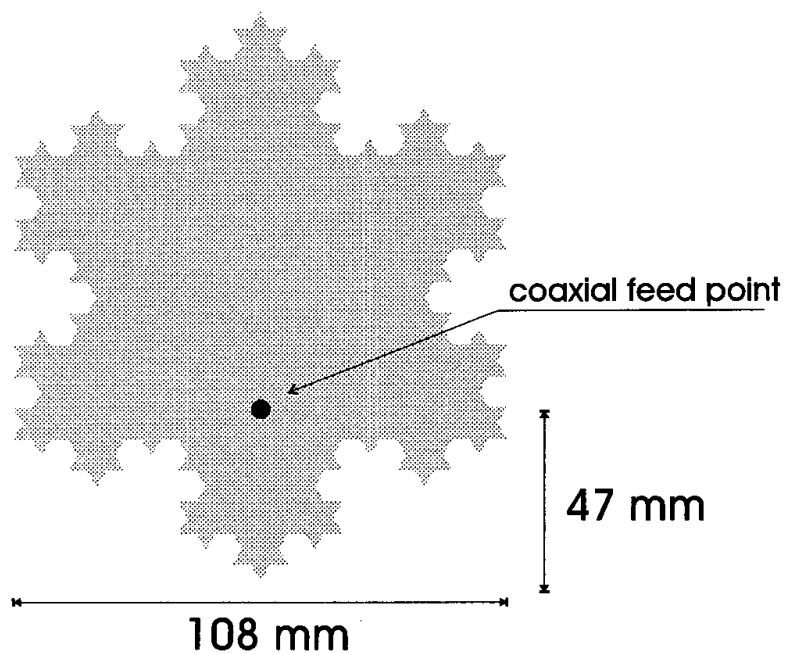

Figure 1: Koch island patch antenna. The antenna has been etched on dielectric substrate. The height of the patch above the ground plane is $3.5 \mathrm{~mm}$. A coaxial feed has been used.
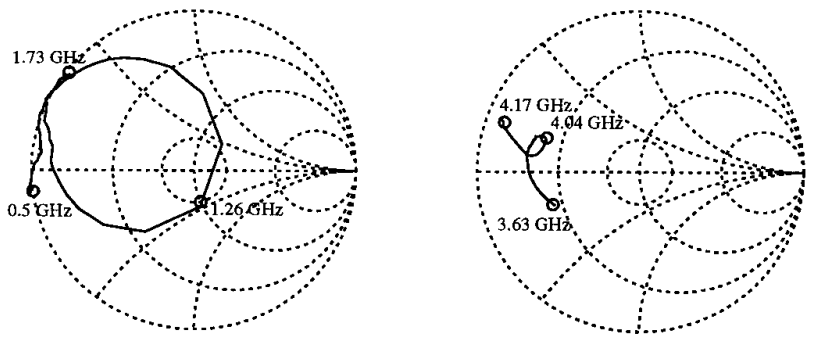

Figure 2: Input impedance of the Koch island patch antenna at the fundamental resonant frequency (left) and at a higher localized mode (right). 


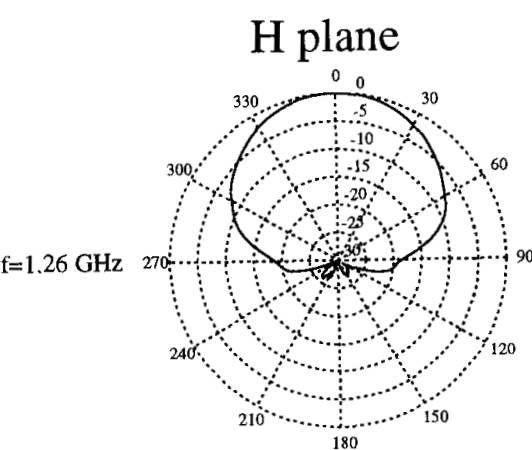

(a)

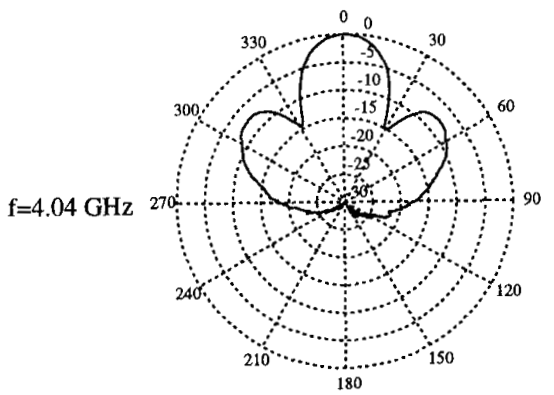

(c)

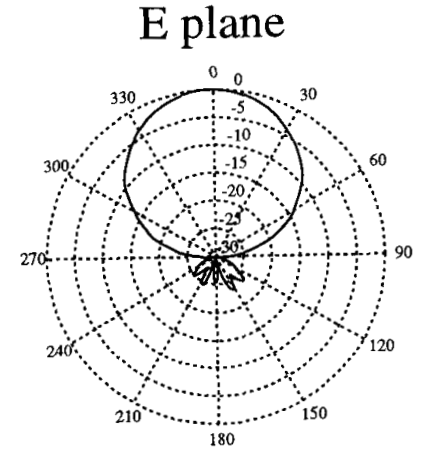

(b)

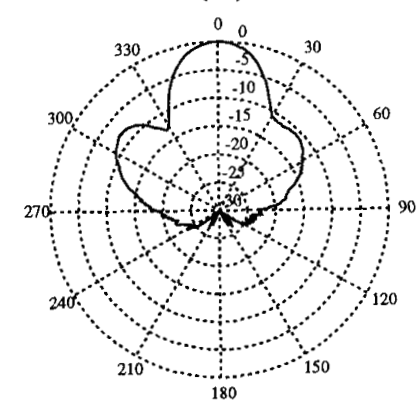

(d)

Figure 3: Radiation pattern for the Koch island patch antenna. Plots (a) and (b) show the main cuts at the fundamental frequency, and (c) and (d) a higher resonant frequency. The localized fractino at this frequency results in a more directive pattern. 